\title{
X-RAY OBSERVATIONS OF BLAZARS WITH GINGA AND ASCA
}

\author{
M. TASHIRO, K. MAKISHIMA and Y. KOHMURA \\ The University of Tokyo, 7-3-1 Hongo, Bunkyo-ku, Tokyo, Japan \\ T. OHASHI \\ Tokyo Mitropolitan University, 1-1 Minami-Osawa, Hachioji, Tokyo, Japan \\ C. OTANI, T. KII, R. FUJIMOTO and F. MAKINO \\ Institute of Space and Astronautical Science, 3-1-1 Yoshinodai, Sagamihara, Kanagawa, Japan \\ and \\ GINGA TEAM and ASCA TEAM
}

\section{Ginga Observations of Highly Variable BL Lacs}

Among 13 BL Lacs observed with Ginga, 1H 0323+022, Mkn 421 and PKS 2155304 exhibited significant variablity during each (typically one day) observation [1]. On the flux-hardness plane, the data points obtained from each source draw a sort of clockwise hysteresis motion. It means that the spectrum hardens before the source gets brighter, while the spectrum softens before the source becomes fainter. Such a soft-lag behavior, first pointed out for PKS2155-304 by Sembay et al. [2]. These properties were also confirmed with the discrete cross correlation function technique.

The soft-lag property is predicted by the synchrotron emission model. Suppose that synchrotron emission arises from relativistic electrons, continuously injected with a fixed spectral shape into a region of homogeneous magnetic field. In this case, flux of the more energetic electrons are expected to respond more quickly to the changes in the electron injection rate because they have shorter life times, thus producing the larger time lags for the softer X-rays.

\section{2. $A S C A$ Observation of Mkn 421}

We observed Mkn 421 on May 10-11, 1993 as one PV observations of $A S C A$. The source was in a low state on 10 and flared up on 11. Using PHA ratio technique, we confirmed that convex or flat spectrum is exhibited in the low state, while flat or concave spectrum in the flaring state. Although the results are still preliminary, it can support not only the similar results obtained by Tashiro with Ginga [1], but also the synchrotron emission model mentioned above.

\section{References}

1. Tashiro, M.: 1993, Ph. D. Thesis, the University of Tokyo

2. Sembay, S. et al. : 1993, ApJ 404, pp. 112-123

T. J.-L. Courvoisier and A. Blecha: Multi-Wavelength Continuum Emission of AGN, 383.

(C) 1994 IAU. Printed in the Netherlands. 